\title{
CONTROL FOR RECYCLE SYSTEMS BASED \\ ON A DISCRETE TIME MODEL APPROXIMATION ${ }^{1}$
}

\author{
B. del-Muro-Cuellar* M. Velasco-Villa** \\ H. Puebla * and J. Alvarez-Ramirez * \\ * Instituto Mexicano del Pétroleo, PIMAyC. \\ Apartado Postal 07730, México DF, México \\ \{bdelmuro,hpuebla\}@imp.mx,jjr@xanum.uam.mx \\ ** CINVESTAV-IPN \\ Departamento de Ingeniería Eléctrica \\ Sección de Mecatrónica \\ A.P. 14-740, 07000, México DF, México \\ velasco@cinvestav.mx.
}

\begin{abstract}
Exponential polynomials arising in transfer functions of chemical processes with recycle and time-delay preclude the use of standar control technics designed for free-delay systems. In this work a simple and effective methodology to derive an approximate discrete-time model free of delay of a continuous timedelayed systems describing recycle and dead-time processes is proposed. The method is based on the discretization of a sampled version of the time-delay term in the original continuous model. Copyright (c) 2005 IFAC
\end{abstract}

Keywords: Time delay; Discrete time; Discrete digital dynamic control; Chemical industry; Model approximation.

\section{INTRODUCTION}

Recycle and time-delay systems are founded commonly in chemical processes. On the one hand, recycle systems enable the energy and matter to be recovered in an industrial process. On the other hand, transport delays are intrinsic feature of chemical plants either with or without recycle. Thus, to obtain realistic dynamic behavior of a simulated chemical process, a nonminimum-phase element should be included in the loop, preferably time-delay (Shynskey, 2002). Moreover, typical models of many interconnected reactor and separation units include both recycle and timedelay.

\footnotetext{
1 Partially Supported by CONACyT-México under Grant 42093.
}

The design of the control system for process with recycle and dead-time presents some specific difficulties, because neglecting the effect of the recycle and dead-time leads to unsatisfactory performance and in some cases instabilities may appear in the closed-loop response. Recycle and deadtime systems leads, in general, to transfer functions with quasipolynomials including transcendental exponential terms in both the denominator and the numerator, and consequently the factorization into rational transfer function and pure delay is not possible. These denominator deadtime induced terms preclude the use of standard controller design techniques as the mathematical methods employed by these techniques require transfer functions with rational denominators. Model approximation has been proposed to remove the exponential term from the denominator, 
such as the method of moments (Papadourakis et al., 1989) and Pade approximations (MalekZavarei and Jamshidi, 1987).

Several control techniques have been proposed to control both recycle and dead-time processes. To improve the performance of time-delay systems, special control techniques have been developed, such as the Smith predictor, (Smith, 1957) and internal model control (Morari and Zafiriou, 1989). All of them provide time-delay compensation based on process dynamic models.

In this work, we propose a quite simple methodology to approximate models of systems with recycle and dead-time. The key of the methodology is to introduce a discrete-time model with a sampled virtual input dead-time at the state by a zero order or a triangular hold, which can capture the dynamics of the continuous-time model. The main advantage of the proposed approximation methodology is that a discrete-time model free of time delay is obtained. Then, the control design based on the approximate discrete-time model can be addressed with different compensator designs, such as simple PID controllers and more sophisticated model-based controllers.

The paper is organized as follows. In Section 2, we present a certain class of time-delay systems, which will be used to represent continuous-time models of both processes with dead-time and recycle. In Section 3 the approximate discretetime model is derived. In Section 4, an example taken from the chemical-process control literature with potential application in chemical processes is used to shown the validation of the proposed approximation and the closed-loop behavior of control designs based on the approximate discretetime models. Finally, conclusions are carried out in Section 5.

\section{A CLASS OF TIME-DELAY SYSTEMS}

In this section, we present the class of time delay systems that involve time delays at the input signal and at the state. For typical recycle processes, simple block diagram manipulations result in transfer functions that contain denominator dead-time terms. Thus, recycle processes with time delay in both forward and recycle paths can be considered within this class of invariant time delay systems as shown below.

Consider the following class of linear time-delay systems:

$$
\begin{aligned}
\dot{x}(t) & =A x(t)+A_{1} x(t-h)+B u(t-\tau) \\
y(t) & =C x(t) \\
x(\varphi) & =\phi(\varphi), \varphi \in[-h, 0]
\end{aligned}
$$

where $x \in \mathbb{R}^{n}$ is the state vector, $u \in \mathbb{R}$ is the input, $y \in \mathbb{R}$ is the output, $h \geq 0$ is the time- delay associated to the state, $\tau \geq 0$ is the timedelay associated to the input, $\phi(\varphi)$ is a continuous function of initial conditions with $-h \leq \varphi \leq 0$. Finally, $A, A_{1} \in \mathbb{R}^{n \times n}, B \in \mathbb{R}^{n \times 1}$ and $C \in \mathbb{R}^{1 \times n}$ are matrices and vectors of systems parameters.

For simplicity in the presentation, and without losing generality, we will consider the SISO case systems, taking into account time delays in the state and input. However, the extension of this method to the multivariable case is straightforward. Taking the Laplace transform of the system (1) leads to the following expression:

$$
\begin{aligned}
s X(s) & =\left(A+A_{1} e^{-h s}\right) X(s)+B e^{-\tau s} U(s) \\
Y(s) & =C X(s)
\end{aligned}
$$

which can be re-written as follows:

$$
\frac{Y(s)}{U(s)}=C\left[s I-\left(A+A_{1} e^{-h s}\right)\right]^{-1} B e^{-\tau s}
$$

The characteristic equation of the above equation is described by a quasipolynomial of the form $\operatorname{det}\left(s I-A-A_{1} e^{-h s}\right)=0$. The time-delay associated with the state $h$ leads to an equation with an infinite number of solutions. It is noticed that the class of systems described by (1) can be represented by differential-difference equations.

Remark 2.1. From a theoretical point of view the stability of linear time invariant systems with time delays at the input signal and the state has been widely studied producing several methodologies to evaluate their stability properties. In particular in Mori and Kokame (Mori and Kokame, 1989) and Wang (Wang, 1992), the stability properties of systems described by (1) are analyzed leading to sufficient conditions based on the value of the time delays.

\subsection{Recycle Systems as Time-Delay Systems}

Consider the following recycle system,

$$
Y(s)=\left[G_{f}(s) G_{f}(s) G_{r}(s)\right]\left[\begin{array}{c}
U(s) \\
Y(s)
\end{array}\right]
$$

where $G_{f}(s)=\frac{N_{f}(s) e^{-n s}}{D_{f}(s)}$ and $G_{r}(s)=\frac{N_{r}(s) e^{-h s}}{D_{r}(s)}$, with $G_{f}(s)$ and $G_{r}(s)$ are the transfer function of the forward and recycle path respectively. $N_{*}(s)$ and $D_{*}(s)$ are polynomials in the Laplace complex variable $s, h \geq 0$ is the time-delay of the recycle transfer function $G_{r}(s)$ and $n \geq 0$ is the timedelay of the forward transfer function $G_{f}(s) . U(s)$ and $Y(s)$ are the Laplace transforms of the input and output signals, respectively.

Simple block diagram manipulations give the following open-loop transfer function for the entire plant,

$$
G_{t}(s)=\frac{D_{r}(s) N_{f}(s) e^{-n s}}{D_{f}(s) D_{r}(s)-N_{f}(s) N_{r}(s) e^{-h s} e^{-n s}}
$$




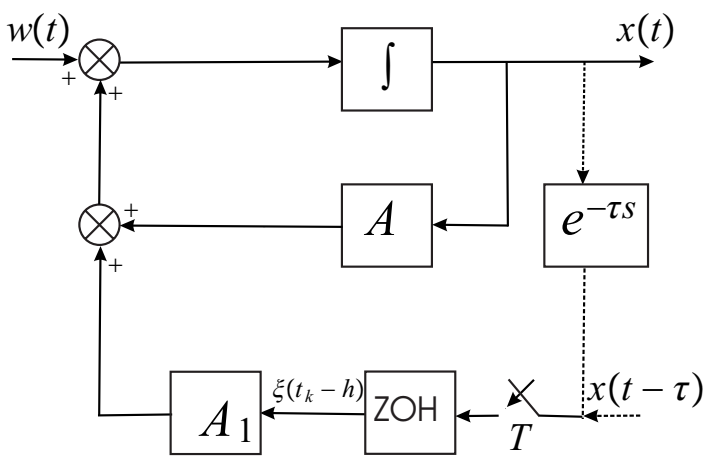

Fig. 1. General scheme of approximation.

The structure is the same as that in Eq. (2) and exponential terms appears explicitly in the denominator and numerator. Note that the corresponding characteristic equation is described by a quasipolynomial equation with a state time delay dependence.

\section{APPROXIMATE DISCRETE-TIME MODEL}

The main purpose of this section is to propose a simple and effective methodology to obtain an approximate discrete time representation of the original continuous time delay systems described by (1).

Let us obtain a sampled time-delay model. We consider the dead-time associated to the state as a second (virtual) input to the system. Introducing a zero order hold $(\mathrm{ZOH})$ to the virtual input, as shown in Figure 1, we have the following partially sampled system:

$$
\begin{aligned}
\dot{x}_{r}(t) & =A x_{r}(t)+A_{1} \xi\left(t_{k}-h\right)+B u(t-\tau) \\
y(t) & =C x_{r}(t) \\
x\left(t_{0}-h\right) & =\phi(\varphi)
\end{aligned}
$$

where $\xi\left(t_{k}-h\right)$ is a piecewise constant function which corresponds to the virtual (sampled) input for system (1).

Theorem 3.1. Consider the system (1) and the partially sampled system (5) (see Figure 1). Under this sampling condition, the solution $x_{r}(t)$ of system (5) is an approximation of the solution $x(t)$ of system (1) with respect to the sampling period $T$.

Proof. Consider the solution of system (1) for $t_{0} \leq t \leq h$ and the initial condition $\xi(t-h)$. Notice that for $t_{0} \leq t \leq h$, the delayed state $x(t-h)$ is determined by the initial condition function and then, it is possible to consider the following systems for $t_{0} \leq t \leq h$ :

$$
\begin{aligned}
\dot{x}(t) & =A x(t)+A_{1} \xi(t-h)+B u(t-\tau) \\
\dot{x}_{r}(t) & =A x_{r}(t)+A_{1} \xi\left(t_{k}-h\right)+B u(t-\tau) .
\end{aligned}
$$

Defining the error signal as $e_{x}(t)=x(t)-x_{r}(t)$, the following dynamical system is obtained:

$$
\dot{e}_{x}(t)=A e_{x}(t)+A_{1} \gamma(t)
$$

where $\gamma(t)$ is a function defined as

$$
\gamma(t)=\xi(t-h)-\xi\left(t_{k}-h\right),
$$

$\gamma(t)$ correspond to the error induced by the consideration of the $\mathrm{ZOH}$ on the delayed state feedback loop of system (1). In order to show explicitly the order of approximation of system (5) with respect to (1) consider also the solution of system (6) on the segment $t_{0} \leq t \leq h$, which is given by

$$
e_{x}(t)=\Phi\left(t, t_{0}\right) e_{x}\left(t_{0}\right)+\int_{t_{0}}^{h} \Phi(t, s) A_{1} \gamma(s) d s
$$

considering the effect of the $\mathrm{ZOH}$ and the fact that the initial condition is the same for systems (1) and (5) it follows that

$$
e_{x}\left(t_{0}\right)=\xi\left(t_{0}\right)-\xi_{r}\left(t_{0}\right)=0
$$

then,

$$
e_{x}(t)=\int_{t_{0}}^{h} \Phi(t, s) A_{1} \gamma(s) d s
$$

Since $\gamma(t)=\xi(t-h)-\xi\left(t_{k}-h\right)$ is a consequence of the $\mathrm{ZOH}$, then $\gamma(t)$ is a function of order one (with respect to the sampling period $T$ ), this is $\gamma(t)=\mathcal{O}(T)$. The equation (7) can be seen as an integration error between functions $\xi(t-h)$ and $\xi\left(t_{k}-h\right)$, therefore, considering a rectangular (by the effect of the $\mathrm{ZOH}$ ) numerical integration method (quadrature formula), (Mathews, 1992) it follows that the integral (7) represents also a function of order one. To conclude the proof it is suffice to consider the step method (Driver, 1977) for the solution of system (6) over the segments $t_{k} \leq t \leq t_{k}+h$.

From the above developments it can be seen that the error $e_{x}(t)$ can be minimized by considering a sampling and hold device of greater order, for example, a first order hold, that correspond in terms of numerical integration to the use of a trapezoidal method. On the other hand, it is important to note that the error of approximation $e_{x}(t)$ depends directly over the sampling period $T$. Therefore, increasing the frequency $T \rightarrow 0$, will also improve the approximation.

Now, let us get the discrete-time model. Consider the solution $x_{r}(t)$ of the (partially sampled) system (5), over the interval $t_{0} \leq t \leq h$, with the sampled initial condition $\xi\left(t_{k}-h\right)$ :

$$
\begin{aligned}
x_{r}(t) & =\Phi\left(t, t_{0}\right) x\left(t_{0}\right)+\int_{t_{0}}^{h} \Phi(t, s) A_{1} \xi\left(t_{k}-h\right) d s \\
& +\int_{t_{0}}^{h} \Phi(t, s) B u(s-\tau) d s .
\end{aligned}
$$

$\xi\left(t_{k}-h\right)$ and $u(t-\tau)$ can be viewed as external inputs. By considering a $\mathrm{ZOH}$ over the input $u(t-$ $\tau)$ it is possible to write 


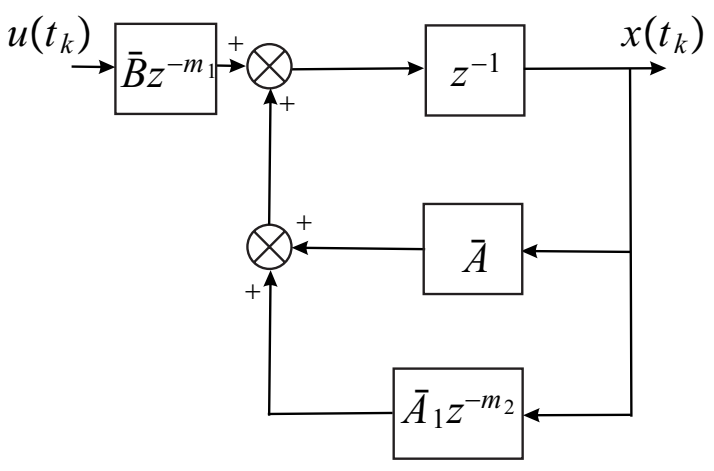

Fig. 2. Approximate discrete time system.

$$
\begin{aligned}
& \begin{array}{l}
x_{r}\left(t_{k+1}\right)= \\
\qquad\left(t_{k+1}, t_{k}\right) x\left(t_{k}\right)+\int_{t_{k}}^{t_{k+1}} \Phi\left(t_{k+1}, s\right) d s \times \\
\times\left[A_{1} \xi\left(t_{k}-h\right)+B u\left(t_{k}-\tau\right)\right]
\end{array} \\
& \text { where } \Phi\left(t_{k+1}, t_{k}\right)=e^{\left(t_{k+1}-t_{k}\right) A}=e^{T A} .
\end{aligned}
$$

Proposition 3.2. An approximate discrete-time model for the continuous-time system (1) is given by

$$
x_{k+1}=\bar{A} x_{k}+\bar{A}_{1} x\left(t_{k}-h\right)+\bar{B} u\left(t_{k}-\tau\right)
$$

with

$$
\begin{aligned}
& \bar{A}=\Phi\left(t_{k+1}, t_{k}\right), \bar{A}_{1}=\int_{t_{k}}^{t_{k+1}} \Phi\left(t_{k+1}, s\right) d s A_{1} \\
& \bar{B}=\int_{t_{k}}^{t_{k+1}} \Phi\left(t_{k+1}, s\right) d s B
\end{aligned}
$$

Proof. From the above developments and from Theorem 3.1 we have that $x_{r}(t)$ is an approximation for $x(t)$, then for $t_{0} \leq t \leq h$, it can be obtained the following approximate discrete-time system

$$
x_{k+1}=\bar{A} x_{k}+\bar{A}_{1} \xi\left(t_{k}-h\right)+\bar{B} u\left(t_{k}-\tau\right)
$$

applying the above developments over the segments $t_{k} \leq t \leq t_{k}+h$, then it follows that the initial condition $\xi\left(t_{k}-h\right)$ are equal to the delayed state $x\left(t_{k}-h\right)$ obtained on the precedent segment of time. This concludes the proof.

It is noticed, considering that the time delays $h$ and $\tau$ of system (1) are multiple of the sampling period $T$, this is $\tau=m_{1} T$ and $h=m_{2} T$, (commensurable delays) system (8) can be depicted as in Figure 2.

Now let us obtain the discrete-time model of Recycle Systems. As stated above, while the transfer functions for the recycle system may be constructed from the individual linear transfer functions for each of the subunits comprising the plant, the resultant overall plant transfer function will often contain denominator dead-time induced terms. It is therefore necessary to simplify these models before they can be used for control analysis and control design purposes in a standard sense. By exploiting the transfer function of the overall recycle systems (4) we have the following result.
Proposition 3.3. An approximate discrete time model for the continuous time recycle system (3) is given by

$$
Y(z)=\left[\begin{array}{lll}
G_{f}(z) & G_{f r}(z)
\end{array}\right]\left[\begin{array}{l}
U(z) \\
Y(z)
\end{array}\right],
$$

where $G_{f}(z)=\left(1-z^{-1}\right) \mathcal{Z}\left(\frac{G_{f}(s)}{s}\right)$ and $G_{f r}(z)=$ $\left(1-z^{-1}\right) \mathcal{Z}\left(\frac{G_{f r}(s)}{s}\right)$, i.e., $G_{f}(z)$ and $G_{f r}(z)$ represent the $z$ transform of $G_{f}(s)$ and $G_{f}(s) G_{r}(s)$ respectively, considering a $\mathrm{ZOH}$ device.

Proof. The proof is direct from the results obtained in the previous section, considering the fictitious (two inputs) system

$$
Y(s)=\left[G_{f}(s) G_{f}(s) G_{r}(s)\right]\left[\begin{array}{c}
U(s) \\
U_{1}(s)
\end{array}\right]
$$

and the corresponding discrete-time system

$$
Y(z)=\left[G_{f}(z) G_{f r}(z)\right]\left[\begin{array}{c}
U(z) \\
U_{1}(z)
\end{array}\right]
$$

where $G_{f}(z)$ and $G_{f r}(z)$ represent the $z$ transform of $G_{f}(z)$ and $G_{f}(s) G_{r}(s)$, respectively, considering a $\mathrm{ZOH}$ at the inputs.

From (9), the global transfer function for the approximate discrete-time model of recycle systems with dead-time can be obtained as

$$
G_{t}(z)=\frac{D_{f r}(z) N_{f}(z)}{D_{f}(z) D_{f r}(z)-D_{f}(z) N_{f r}(z)}
$$

with $N_{f}(z), N_{f r}(z), D_{f}(z)$ and $D_{f r}(z)$ are polynomials in $z$ such that $G_{f}(z)=\frac{N_{f}(z)}{D_{f}(z)}$ and $G_{f r}(z)=$ $\frac{N_{f r}(z)}{D_{f r}(z)}$.

Once the transfer function of the approximate discrete model $G_{t}(z)=R(z) / W(z)$ has been computed, a feedback control design based on the approximate discrete time model can be addressed with different compensator designs, such as simple PI controllers, standard polynomial approaches, and more sophisticated model-based controllers. This will be illustrated in the following section with an example.

\section{EXAMPLE}

A case study consisting of a chemical engineering prototype recycle system with dead-time in both the forward and recycle path, will be used to demonstrate the steps of the methodology and the closed-loop performance of a discrete time control design based on a polynomial approach (del MuroCuellar and Alvarez-Ramirez, 2003).

We have taken a recycle system and consisting of two units: $G_{f}(s)$ in the forward path and $G_{r}(s)$ in 
the recycle path. Each unit is a time-delay linear system with the following transfer functions:

$$
G_{f}(s)=\frac{e^{-0.4 s}}{s+1}, G_{r}(s)=\frac{e^{-0.2 s}}{s+1}
$$

Then, the description of the system is given by

$$
\begin{aligned}
Y(s) & =\left[G_{f}(s) G_{f}(s) G_{r}(s)\right]\left[\begin{array}{l}
U(s) \\
Y(s)
\end{array}\right] \\
& =\left[\frac{e^{-0.4 s}}{s+1} \frac{e^{-0.6 s}}{(s+1)^{2}}\right]\left[\begin{array}{l}
U(s) \\
Y(s)
\end{array}\right]
\end{aligned}
$$

or equivalently,

$$
\frac{Y(s)}{U(s)}=\frac{(s+1) e^{-0.4 s}}{(s+1)^{2}-e^{-0.2 s}}
$$

where $Y(s)$ is the output and $U(s)$ is the input.

Let us consider the fictitious (two inputs) system

$$
Y(s)=\left[\begin{array}{ll}
\frac{e^{-0.4 s}}{s+1} & \frac{e^{-0.6 s}}{(s+1)^{2}}
\end{array}\right]\left[\begin{array}{c}
U(s) \\
U_{1}(s)
\end{array}\right]
$$

The corresponding discrete system with a $\mathrm{ZOH}$ at the inputs and a sampling period $T=0.2$, is given by,

$$
\begin{aligned}
Y(z)= & {\left[\frac{0.183}{z^{3}-0.818 z^{2}} \frac{0.01752 z+0.01534}{z^{5}-1.637 z^{4}+0.670 z^{3}}\right] } \\
& \times\left[\begin{array}{c}
U(z) \\
U_{1}(z)
\end{array}\right]
\end{aligned}
$$

The above system is a discrete-time approximation of the continuous system (10). By considering $U_{1}(z)=Y(z)$ we get the approximate discretetime model for system (10), based on a $\mathrm{ZOH}$,

$$
\begin{aligned}
Y(z) & =\left[\begin{array}{ll}
\frac{0.183}{z^{3}-0.818 z^{2}} & 0.0175 z+0.0153 \\
z^{5}-1.637 z^{4}+0.670 z^{3}
\end{array}\right] \\
& \times\left[\begin{array}{l}
U(z) \\
Y(z)
\end{array}\right]
\end{aligned}
$$

The accuracy of the approximation can be improved by using a triangular hold $(\mathrm{TH})$ at the input $U_{1}(z)$ (with a $\mathrm{ZOH}$ at the input $U(z)$ ). Following the procedure as above we get,

$$
\begin{aligned}
& Y(z)= \\
& {\left[\frac{0.183}{z^{3}-0.8187 z^{2}} \frac{0.0060 z^{2}+0.0218 z+0.0049}{z^{5}-1.637 z^{4}+0.6703 z^{3}}\right]} \\
& \times\left[\begin{array}{l}
U(z) \\
Y(z)
\end{array}\right]
\end{aligned}
$$

We can use the approximate discrete-time model based on the triangular hold (11) in order to design a discrete controller to the system (10).

The transfer function (11) can be re-written as,

$$
\frac{Y(z)}{u(z)}=\frac{B(z)}{A(z)}
$$

with

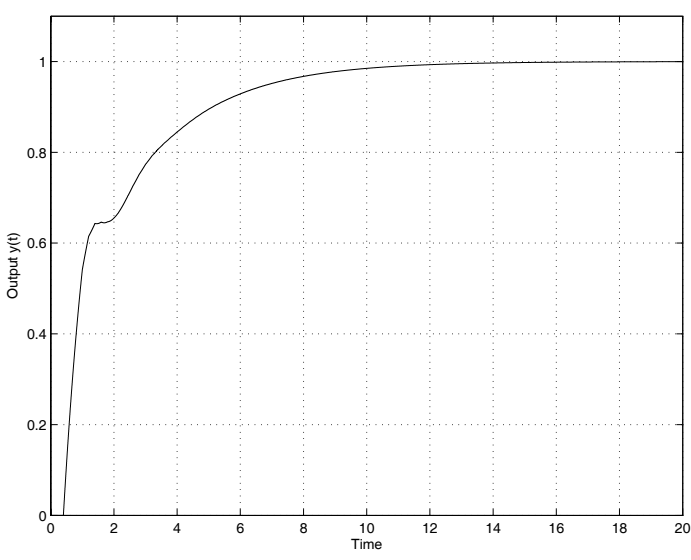

Fig. 3. Output following a step unit input.

$$
\begin{aligned}
B(z) & =0.1813 z^{3}-0.2968 z^{2}+0.1215 z \\
A(z) & =z^{6}-2.456 z^{5}+2.011 z^{4}-0.5548 z^{3} \\
& -0.0169 z^{2}+0.0129 z+0.00404
\end{aligned}
$$

It can be seen from (12), that the discrete-time model has a pole in $z=-1$. Then, if the discrete model is adequate, a simple proportional controller can follows step inputs. This property is illustrated in Figure 3 on the continuous system, with the control input $u(k T)=1.2[v(k T)-y(k T)]$ and $v(k T)=1$.

Based on the approximate discrete-time model we design a pole-placement discrete controller (del Muro-Cuellar and Alvarez-Ramirez, 2003), in order to shown the potential applications of the approximation methodology for control design purposes. This methodology provides a two degree of freedom controller that allows to follow step reference inputs and to reject step disturbances at the input.

The discrete-time controller has the following structure: $U(z)=\left[\frac{R(z)}{S(z)} Y_{\text {ref }}(z)-\frac{R(z)}{S(z)} Y(z)\right]$, where $Y_{\text {ref }}(z)$ is the reference command and $R(z), T(z)$ and $S(z)$ are polynomials in the complex variable $z$. This control structure is equivalent to an estimated (complete) state feedback procedure endowed with integral action.

The characteristic polynomial $D(z)$ induced by the controller is

$$
D(z)=A(z) S(z)+B(z) R(z)
$$

In order to obtain the controller, the characteristic polynomial $D(z)$ must be defined. To reject external (step) loads, the polynomial $S(z)$ should contain the factor $(z-1)$, i.e., $S(z)=(z-1) S^{\prime}(z)$. In order to obtain proper transfer functions $\frac{R(z)}{S(z)}$ and $\frac{T(z)}{S(z)}$ and a unique solution to the equation (13), the degree of $D(z)$ must be $2 n$, with $n$ the order of $G_{t}(z)$. 


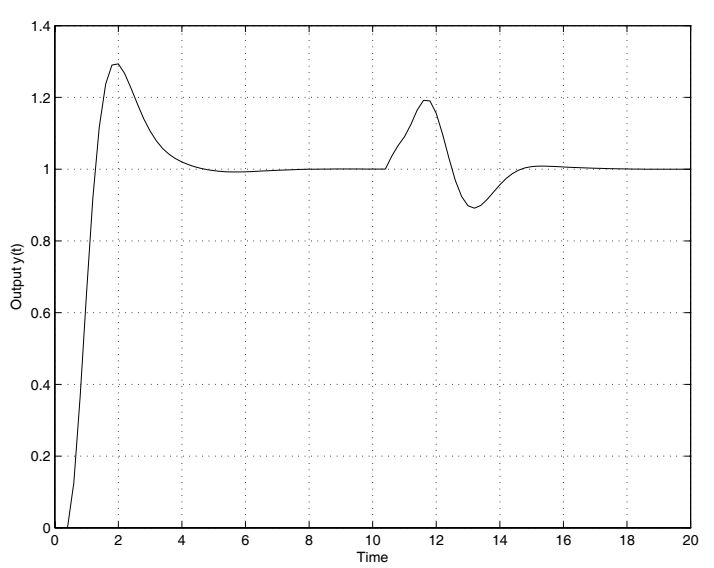

Fig. 4. Output following step input and rejecting step disturbance for example 1.

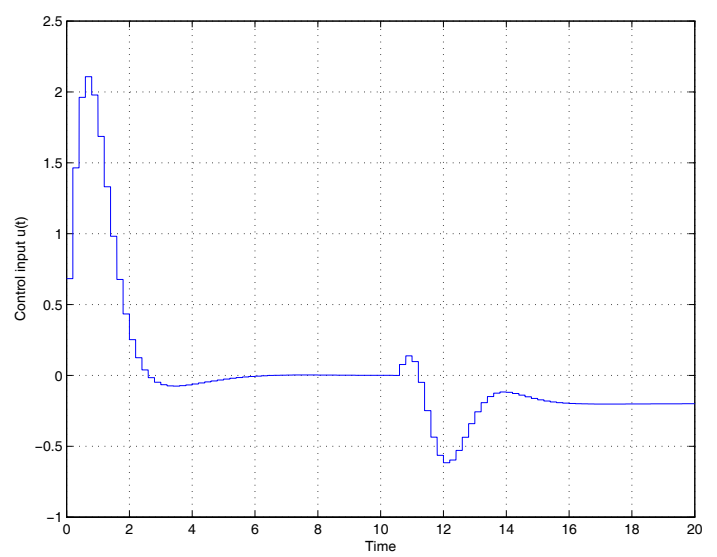

Fig. 5. Control input $u(t)$ for example 1.

From the approximate discrete-time model (12) we can get the following discrete controller:

$$
\begin{aligned}
T(z) & =0.6827 z^{6}-2.4576 z^{5}+3.6864 z^{4}-2.9491 z^{3} \\
& +1.3271 z^{2}-0.3185 z+0.0319 \\
R(z) & =-2.1112 z^{6}+10.0611 z^{5}-19.0533 z^{4} \\
& +18.4915 z^{3}-9.7678 z^{2}+2.6862 z-0.3037 \\
S(z) & =z^{6}-4.7440 z^{5}+10.0977 z^{4}-12.2422 z^{3} \\
& +8.7128 z^{2}-3.3621 z+0.5377
\end{aligned}
$$

This discrete-time controller places all the closedloop poles at $z=0.6$. Figure 4 shows the performance of the closed-loop system under a unit step input and a step disturbance (20\%) applied at $t=10 \mathrm{sec}$. In a short-time scale, $5 \mathrm{~s}$, the system follows the step input and rejects the step input disturbance. Figure 5 shows the discrete control input $u(t)$, which is acceptable for practical implementation purposes.

\section{CONCLUSIONS}

We have proposed a model approximation methodology with applications to dead-time and recycle systems. Recycle processes with time delay in both forward and recycle paths can be considered within a general class of invariant systems involving time delays at the input signal and at the state. By means of either a zero order or a triangular hold on the time-delay at the state we have derived a sampled time-delay model which is used to obtain an approximate discrete-time model free of dead-time of an original time-delay continuous model. Using the presented method, we can choose arbitrarily a suitable approximation of a delay element for a desirable design. Although the suggested approach is restricted to the class of recycle systems described here, it can be applied to a wide class of linear systems including non-minimum phase systems. Numerical simulations on a recycle system with dead-time in both the forward and direct paths show both the effectiveness of the proposed model approximation methodology and the good closed-loop behavior of control designs based on approximate discretetime plant model.

\section{REFERENCES}

del Muro-Cuellar, B. and J. Alvarez-Ramirez (2003). A note on high-order controllers for time-delay processes. Ind. Eng. Chem. Res. 42, 4992-5002.

Driver, R. D. (1977). Ordinary and delay differential equations. Springer-Verlag. London.

Malek-Zavarei, M. and M. Jamshidi (1987). TimeDelay systems. Analysis, optimization and applications. NorthHolland. The Netherlands.

Mathews, J. H. (1992). Numerical methods for mathematics science and engineering. Prentice-Hall. New York.

Morari, M. and E. Zafiriou (1989). Robust Process Control. Prentice-Hall, Englewood Cliffs, New Jersey.

Mori, T. and H. Kokame (1989). Stability of $\dot{x}(t)=a x(t)+b x(t-\tau)$. IEEE Transactions on Automatic Control 34, 460-462.

Papadourakis, A., M. F. Doherty and J. M. Douglas (1989). Approximate dynamic models for chemical process systems. Ind. Eng. Chem. Res. 28, 546-552.

Shynskey, F. G. (2002). Process control: as taught vs as practiced. Ind. Eng. Chem. Res. 28, 546-552.

Smith, O. J. M. (1957). Closer control of loops with deadtime. Chem. Eng. Prog. 53(5), 217219.

Wang, S. S. (1992). Further results on stability of $\dot{x}(t)=a x(t)+b x(t-\tau)$. System and Control Letters 19, 165-168. 